\title{
PSEUDO-UMBILICAL SURFACES IN EUCLIDEAN SPACES
}

\author{
By BANG-Yen Chen
}

Recently, the author introduced the notion of $\alpha$ th curvatures of first and second kinds for surfaces in higher dimensional euclidean space $[2,3]$. The main purpose of this paper is to study these curvatures more detail. In $\S 1$, we derive some integral formulas for the $\alpha$ th curvatures of first and second kinds. In $\S 2$, we get some applications of these formulas to pseudo-umbilical surfaces.

\section{$\S 1$. Integral formulas for ath curvatures.}

Let $M^{2}$ be an oriented closed Riemannian surface with an isometric immersion $x: M^{2} \rightarrow E^{2+N}$. Let $F\left(M^{2}\right)$ and $F\left(E^{2+N}\right)$ be the bundles of orthonormal frames of $M^{2}$ and $E^{2+N}$ respectively. Let $B$ be the set of elements $b=\left(p, e_{1}, e_{2}, \cdots, e_{2+N}\right)$ such that $\left(p, e_{1}, e_{2}\right) \in F\left(M^{2}\right)$ and $\left(x(p), e_{1}, \cdots, e_{2+N}\right) \in F\left(E^{2+N}\right)$ whose orientation is coherent with that of $E^{2+N}$, identifying $e_{i}$ with $d x\left(e_{i}\right), i=1,2$. Then $B \rightarrow M^{2}$ may be considered as a principal bundle with fibre $O(2) \times S O(N)$ and $\tilde{x}: B \rightarrow F\left(E^{2+N}\right)$ is naturally defined by $\tilde{x}(b)=\left(x(p), e_{1}, \cdots, e_{2+N}\right)$.

The structure equations of $E^{2+N}$ are given by

$$
d x=\sum_{A} \omega_{A}^{\prime} e_{A}, \quad d e_{A}=\sum_{B} \omega_{A B}^{\prime} e_{B}
$$

(1)

$$
\begin{array}{r}
d \omega_{B}^{\prime}=\sum_{B} \omega_{B}^{\prime} \wedge \omega_{B A}^{\prime}, \quad d \omega_{A B}^{\prime}=\sum_{C} \omega_{A C}^{\prime} \wedge \omega_{C B}^{\prime}, \quad \omega_{A B}^{\prime}+\omega_{B A}^{\prime}=0, \\
A, B, C, \cdots=1,2, \cdots, 2+N,
\end{array}
$$

where $\omega_{A}^{\prime}, \omega_{A B}^{\prime}$ are differential 1-forms on $F\left(E^{2+N}\right)$. Let $\omega_{A}, \omega_{A B}$ be the induced 1forms on $B$ from $\omega_{A}^{\prime}, \omega_{A B}^{\prime}$ by the mapping $\tilde{x}$. Then we have

$$
\begin{aligned}
\omega_{r}=0, & \omega_{i r}=\sum_{\jmath} A_{r i j} \omega_{\jmath}, \\
& A_{r \imath \jmath}=A_{r j i}, \\
i, j, \cdots=1,2 ; & r, t, \cdots=3, \cdots, 2+N .
\end{aligned}
$$

From (1), we get

$$
d \omega_{i}=\sum_{\jmath} \omega_{\jmath} \wedge \omega_{j i}, \quad d \omega_{A B}=\sum_{C} \omega_{A C} \wedge \omega_{C B}
$$

Recelved November 2, 1970. 
Let $\left(p, e_{1}, e_{2}, \bar{e}_{3}(p), \cdots, \bar{e}_{2+N}(p)\right), p \in U$, be a local cross-section of $B \rightarrow F\left(M^{2}\right)$ and for any unit normal vector $e$ at $x(p), p \in U$, put $e=e_{2+N}=\sum \xi_{r} \bar{e}_{r}(p)$. Denoting the restriction of $A_{r \imath \jmath}$ onto the image of this local cross-section by $\bar{A}_{r i j}$, we may put

$$
A_{2+N i \jmath}=\sum_{r} \xi_{r} \bar{A}_{r \imath \jmath}
$$

Hence the Lipschitz-Killing curvature $G(p, e)$ is given by

$$
G(p, e)=\operatorname{det}\left(A_{2+N i j}\right)=\left(\sum_{r} \xi_{r} \bar{A}_{r 11}\right)\left(\sum_{s} \xi_{s} \bar{A}_{s 22}\right)-\left(\sum_{t} \xi_{t} \bar{A}_{t 12}\right)^{2} .
$$

The right hand sides is a quadratic form of $\xi_{3}, \cdots, \xi_{2+N}$. Hence, choosing a suitable cross-section, we can write $G(p, e)$ as

$$
G(p, e)=\sum_{\alpha=1}^{N} \lambda_{\alpha}(p) \xi_{\alpha+2} \xi_{\alpha+2}, \quad \lambda_{1} \geqq \lambda_{2} \geqq \cdots \geqq \lambda_{N} .
$$

We call this local cross-section of $B \rightarrow F\left(M^{2}\right)$, the Frenet cross-section in the sense of $\bar{O} t s u k i$, and the frame $\left(p, e_{1}, e_{2}, \bar{e}_{3}, \cdots, \bar{e}_{2+N}\right)$ the $\bar{O} t s u k i$ frame. We call the curvature $\lambda_{\alpha}$, the $\alpha$ th curvature of the second kind $[2,5]$. By means of the method of definitions, $\lambda_{\alpha}$ are defined continuously on the whole manifold $M^{2}$, and the Ōtsuki frame is defined uniquely on the subset in which $\lambda_{1}>\lambda_{2}>\cdots>\lambda_{N}$. With respect to the Ōtsuki frame the curvatures:

$$
\mu_{\alpha}(p)=\frac{1}{2} \operatorname{trace}\left(\bar{A}_{2+\alpha \imath j}\right), \quad \alpha=1, \cdots, N
$$

are called the $\alpha$ th curvatures of the first kind [2].

With respect to the Ōtsuki frame, we have

$$
\begin{aligned}
& \omega_{1 r} \wedge \omega_{2 r}=\lambda_{r-2} d V, \quad d V=\omega_{1} \wedge \omega_{2}, \quad r=3, \cdots, 2+N, \\
& \omega_{1 r} \wedge \omega_{2 t}+\omega_{1 t} \wedge \omega_{2 r}=0, \quad r \neq t ; \quad r, t=3, \cdots, 2+N .
\end{aligned}
$$

In the following, by a spherical immersion $\bar{x}: M^{n} \rightarrow E^{n+N}$ of a manifold $M^{n}$ into a euclidean $(n+N)$-space $E^{n+N}$ we mean that $M^{n}$ is immersed into a hypersphere of $E^{n+N}$ centered at the origin of $E^{n+N}$ by the immersion $\bar{x}$. Let $X(p)$ denote the position vector of $x(p)$ in $E^{2+N}$ with respect to the origin of $E^{2+N},[, \cdots \cdots$,$] the$ combined operation of exterior product and vector product in $E^{2+N}$, and $(\stackrel{2 \text { terms }}{,})$ the combined operation of exterior product and scalar product in $E^{2+N}$. Then, with respect to the Ōtsuki frame $\left(p, e_{1}, e_{2}, \bar{e}_{3}, \cdots, \bar{e}_{2+N}\right)$, we have

$$
\begin{aligned}
& d\left(X,\left[d \bar{e}_{r}, \bar{e}_{3}, \cdots, \bar{e}_{2+N}\right]\right) \\
= & \left(d X,\left[d \bar{e}_{r}, \bar{e}_{3}, \cdots, \bar{e}_{2+N}\right]\right)-\sum_{s}\left(X,\left[d \bar{e}_{r}, \bar{e}_{3}, \cdots, \bar{e}_{s-1}, d \bar{e}_{s}, \bar{e}_{s+1}, \cdots, \bar{e}_{2+N}\right]\right)
\end{aligned}
$$




$$
\begin{aligned}
= & 2(-1)^{N}\left\{\lambda_{r-2}\left(X \cdot \bar{e}_{r}\right) d V+\mu_{r-2} d V+\frac{1}{2} \sum_{s \neq r}\left(X \cdot \bar{e}_{s}\right)\left(\omega_{1 r} \wedge \omega_{2 s}+\omega_{1 s} \wedge \omega_{2 r}\right)\right. \\
& \left.+\frac{1}{2} \sum_{s \neq r} \omega_{r s} \wedge\left(\left(X \cdot e_{1}\right) \omega_{2 s}-\left(X \cdot e_{2}\right) \omega_{1 s}\right)\right\} \\
= & 2(-1)^{N}\left\{\left(X \cdot \bar{e}_{r}\right) \lambda_{r-2} d V+\mu_{r-2} d V+\sum_{s \neq r} \frac{1}{2} \omega_{r s} \wedge\left(\left(X \cdot e_{1}\right) \omega_{2 s}-\left(X \cdot e_{2}\right) \omega_{1 s}\right)\right\} .
\end{aligned}
$$

Suppose that $x: M^{2} \rightarrow E^{2+N}$ is spherical. Then the last term of the above equations vanishes. Hence, we get

$$
d\left(X,\left[d \bar{e}_{r}, \bar{e}_{3}, \cdots, \bar{e}_{2+N}\right]\right)=2(-1)^{N}\left\{\left(X \cdot \bar{e}_{r}\right) \lambda_{r-2}+\mu_{r-2}\right\} d V .
$$

Furthermore, suppose that there exist a unit normal vector field $e$ defined globally on $M^{2}$ and a fixed integer $\alpha ; 1 \leqq \alpha \leqq N$, such that the Lipschitz-Killing curvature $G(p, e)=\lambda_{\alpha}(p)$ for all $p \in M^{2}$, then, by the definition of the Ötsuki frame, we know that for each point $p$, there exists an Ōtsuki frame $\left(q, e_{1}, e_{2}, \bar{e}_{3}, \cdots, \bar{e}_{2+N}\right)$ with $\bar{e}_{2+\alpha}=e$ on a neighborhood of $p$. Therefore, by the fact that

$$
d\left(X,\left[d \bar{e}_{r}, \bar{e}_{3}, \cdots, \bar{e}_{2+N}\right]\right)=d\left(X,\left[d \tilde{e}_{r}, \tilde{e}_{3}, \cdots, \tilde{e}_{2+N}\right]\right)
$$

for any two Ōtsuki frames $\left(p, e_{1}, e_{2}, \bar{e}_{3}, \cdots, \bar{e}_{2+N}\right)$ and $\left(p, e_{1}^{\prime}, e_{2}^{\prime}, \tilde{e}_{3}, \cdots, \tilde{e}_{2+N}\right)$ with $\bar{e}_{r}=\tilde{e}_{r}$, we have the following theorem:

THEOREM 1. Let $x: M^{2} \rightarrow E^{2+N}$ be a spherical immersion of an oriented closed surface $M^{2}$ into $E^{2+N}$. If there exist a unit normal vector field $e$ over $M^{2}$ and a fixed integer $\alpha, 1 \leqq \alpha \leqq N$, such that the Lipschitz-Killing curvature $G(p, e)=\lambda_{a}(p)$ for all $p \in M^{2}$, then we have

$$
-\int_{M} \mu_{\alpha} d V=\int_{M^{2}}(X \cdot e) \lambda_{\alpha} d V
$$

where $\mu_{\alpha}$ is the $\alpha$ th curvature of first kind corresponding to $e$.

Furthermore, we have

$$
\begin{aligned}
& d\left(X,\left[d X, \bar{e}_{3}, \cdots, \bar{e}_{2+N}\right]\right) \\
= & \left(d X,\left[d X, \bar{e}_{3}, \cdots, \bar{e}_{2+N}\right]\right)-\sum_{r}\left(X,\left[d X, \bar{e}_{3}, \cdots, \bar{e}_{r-1}, d \dot{e}_{r}, \bar{e}_{r+1}, \cdots, \bar{e}_{2+N}\right]\right) \\
= & 2(-1)^{N-1}\left(1+\sum_{r}\left(X \cdot \bar{e}_{r}\right) \mu_{r-2}\right) d V .
\end{aligned}
$$

Hence, if we define the mean curvature vector $H$ by

$$
H=\sum_{r} \mu_{r-2} \bar{e}_{r}
$$

then, by integrating both sides of (11) over $M^{2}$ and applying the Stokes theorem, we get 
Lemma 2. Let $x: M^{2} \rightarrow E^{2+N}$ be an immersion of an oriented closed surface $M^{2}$ into $E^{2+N}$. Then we have

$$
\int_{M^{2}} d V=-\int_{M^{2}}(X \cdot H) d V
$$

In theorem 1 , if $e$ is parallel to the mean curvature vector and $\alpha=1$, then we have the following corollary:

CoRollary 1. Let $x: M^{2} \rightarrow E^{2+N}$ be a spherical immersion of an oriented closed surface $M^{2}$ into $E^{2+N}$. If the Lipschitz-Killing curvature in the direction of mean curvature vector is nowhere negative and the mean curvature normal is nowhere zero, then we have

$$
-\int_{M^{2}} h d V=\int_{M^{2}}(X \cdot e) \lambda_{1} d V
$$

where $H=h e$.

Proof. If the mean curvature vector $H \neq 0$ everywhere and the LipschitzKilling curvature in the direction of $H$ is nowhere negative, then we can easily verify that $G(p, e)=\lambda_{1}(p)$ for all $p \in M^{2}$. Therefore, by theorem 1 , we get the above integral formula.

\section{§2. Pseudo-umbilical surfaces in Euclidean spaces.}

In [4], the author proved that

LEMMA 3. Let $x: M^{n} \rightarrow E^{n+N}$ be an immersion of an oriented closed n-dimensional manifold $M^{n}$ into $E^{n+N}$. Then the immersion $x$ is spherical if and only if $X \cdot H=-1$.

An immersion $x: M^{n} \rightarrow E^{n+N}$ is called minimal if the mean curvature vector $H=0$ everywhere. If the mean curvature vector $H$ is nowhere vanished, then we can let $e$ be a unit normal vector field in the direction of $H$. In this case, if the second fundamental form in the direction $e, \mathrm{II}_{e}=-d X \cdot d e$, is propotional to the first fundamental form, $\mathrm{I}=d X \cdot d X$, everywhere, then the immersion $x: M^{n} \rightarrow E^{n+N}$ is called pseudo-umbilical [6].

The main purpose of this section is to prove the following:

THEOREM 4. Let $x: M^{2} \rightarrow E^{2+N}$ be a spherical immersion of an oriented closed surface $M^{2}$ into $E^{2+N}$ with the mean curvature vector $H$ nowhere zero. Then the immersion $x$ is pseudo-umbilical if and only if the Lipschitz-Killing curvature in the direction $H=$ he is maximal, i.e. $G(p, e)=\lambda_{1}(p)$ for all $p \in M^{2}$.

Proof. If the immersion $x$ is pseudo-umbilical, then we can choose a local 
cross-section $\left(p, e_{1}, e_{2}, e_{3}^{\prime}, \cdots, e_{2+N}^{\prime}\right)$ such that $e_{3}^{\prime}=e$ parallel to the mean curvature vector $H$. With respect to this cross-section, we can easily find that

$$
A_{311}^{\prime}=A_{322}^{\prime} \neq 0, \quad A_{411}^{\prime}=-A_{422}^{\prime}, \cdots, A_{2+N 11}^{\prime}=-A_{2+N 22}^{\prime},
$$

where $A_{r i j}^{\prime}$ is the restriction of $A_{r \imath \jmath}$ onto the image of this local cross-section. Moreover, by a suitable choosing of $e_{1}, e_{2}$, we can assume that

$$
A_{312}^{\prime}=A_{321}^{\prime}=0 \text {. }
$$

Thus, the Lipschitz-Killing curvature $G(p, e)=\left(A_{311}^{\prime}\right)^{2}>0$ in the direction of mean curvature vector $H=h e$ and the Lipschitz-Killing curvatures $G\left(p, e_{r}^{\prime}\right) \leqq 0$ for all $r=4, \cdots, 2+N$. From these results, we can easily verify that $G(p, e)=\lambda_{1}(p)$ for all $p \in M^{2}$.

Conversely, if the Lipschitz-Killing curvature $G(p, e)$ in the direction of mean curvature vector $H=h e$ is equal to the first curvature of second kind $\lambda_{1}(p)$ everywhere, then, by Corollary 1 , we have

$$
-\int_{M^{2}} h d V=\int_{M^{2}}(X \cdot e) \lambda_{1} d V
$$

Moreover, by the assumption that $x$ is spherical and lemma 3 , we have

$$
h(X \cdot e)=-1 \text {. }
$$

Combining (15) and (16), we get

$$
\int_{M^{2}}\left(\frac{1}{h}\right)\left(h^{2}-\lambda_{1}\right) d V=0
$$

Thus, by the fact $h>0$, and $h^{2}-\lambda_{1} \geqq 0$, we get $h^{2}-\lambda_{1}=0$. From this we can easily prove that the immersion $x$ is pseudo-umbllical. This completes the proof of the theorem.

REMARK 1. If $x: M^{n} \rightarrow E^{n+N}$ is spherical, then we can prove that the mean curvature vector $H \neq 0$ everywhere. So that the condition of $H \neq 0$ everywhere in theorem 4 is not essential.

REMARK 2. In [1, 7], Yano and Chen proved that the only spherical pseudoumbilical submanifolds $M^{n}$ of dimension $n$ in $E^{n+3}$ with constant mean curvature, $h=$ constant, are minimal submanifolds of some hyperspheres of $E^{n+3}$ (not necessary centered at the origin of $E^{n+3}$ ). Thus, by theorem 4 and the Yano-Chen result, we know that if $x: M^{2} \rightarrow E^{5}$ is a spherical immersion of a closed oriented surface in $E^{5}$, then the Lipschitz-Killing curvature in the direction of $H$ satisfies $G(p, e)$ $=\lambda_{1}=$ constant, $H=h e$, when and only when $M^{2}$ is immersed as a minimal surface in some hypersphere of $E^{5}$.

The author would like to express his thanks to Professor T. Ötsuki for his 
valuable suggestion and also thanks to Professor G. D. Ludden for their valuable conversation.

\section{REFERENCES}

[1] Chen, B.-Y., Minımal hypersurfaces in an $m$-sphere. Proc. A.M.S. 29 (1971), 375380.

[2] Chen, B.-Y; On an inequality of T. J. Willmore. Proc. A.M.S. 26 (1970), 473-479.

[3] Chen, B.-Y., On the difference curvature of surfaces in euclidean space. Math. J. Okayama Unıv. 14 (1970), 153-157.

[4] Chen, B.-Y., Submanifolds in a euclidean hypersphere. Proc. A.M.S. 27 (1971), $627-628$.

[5] ŌTsuKI, T., On the total curvature of surfaces in euclidean spaces. Japan. J. Math. 35 (1966), 61-71.

[6] ŌTsuki, T., A theory of Riemannian submanıfolds. Kōdaı Math. Sem. Rep. 20 (1968), 282-295.

[7] Yano, K., AND B.-Y. ChEn, Minımal submanıfolds in a higher dimensional sphere. To appear in Tensor.

Eepartment of Mathematics,

Michigan State University,

East Lansing, Michigan 48823, USA. 